\title{
Integration over an Infinite-Dimensional Banach Space and Probabilistic Applications
}

\author{
Claudio Asci \\ Dipartimento di Matematica e Geoscienze, Università degli Studi di Trieste, Via Valerio 12/1, 34127 Trieste, Italy \\ Correspondence should be addressed to Claudio Asci; casci@units.it
}

Received 27 January 2014; Accepted 18 May 2014; Published 15 June 2014

Academic Editor: Remi Léandre

Copyright (C) 2014 Claudio Asci. This is an open access article distributed under the Creative Commons Attribution License, which permits unrestricted use, distribution, and reproduction in any medium, provided the original work is properly cited.

We study, for some subsets $I$ of $\mathbf{N}^{*}$, the Banach space $E$ of bounded real sequences $\left\{x_{n}\right\}_{n \in I}$. For any integer $k$, we introduce a measure over $(E, \mathscr{B}(E))$ that generalizes the $k$-dimensional Lebesgue measure; consequently, also a theory of integration is defined. The main result of our paper is a change of variables' formula for the integration.

\section{Introduction}

In the mathematical literature, some articles introduced infinite-dimensional measures analogous to the Lebesgue one (see, e.g., the paper of Léandre [1], in the context of the noncommutative geometry, that one of Tsilevich et al. [2], which studies a family of $\sigma$-finite measures on $\mathbf{R}^{+}$, and that one of Baker [3], which defines a measure on $\mathbf{R}^{\mathbf{N}^{*}}$ that is not $\sigma$-finite).

The motivation of this paper follows from the natural extension to the infinite-dimensional case of the results of the article [4], where we estimate the rate of convergence of some Markov chains in $[0, p)^{k}$ to a uniform random vector. In order to consider the analogue random elements in $[0, p)^{\mathbf{N}^{*}}$, it is necessary to overcome some difficulties, for example, the lack of a change of variables' formula for the integration in the subsets of $\mathbf{R}^{\mathbf{N}^{*}}$. A related problem is studied in the paper of Accardi et al. [5], where the authors describe the transformations of generalized measures on locally convex spaces under smooth transformations of these spaces.

In our paper, we consider some subsets $I$ of $\mathbf{N}^{*}$, and we suppose that $\mathbf{R}^{I}$ is endowed with the standard infinity-norm generalized to assume the values in $[0,+\infty]$; then, the vector space $E$ of the elements of $\mathbf{R}^{I}$ with finite norm is a Banach space with respect to the distance defined by the norm. Observe that although in general it is possible to construct a $\sigma$-algebra on $\mathbf{R}^{I}$ simply by considering the product indexed by $I$ of the same Borel $\sigma$-algebra on $\mathbf{R}$, in this way a product of $\sigma$-finite measures $\mu$ on $\mathbf{R}$ can be defined only if $I$ is finite or $\mu$ is a probability measure (by Jessen theorem).

To solve this problem and others, in Section 2 we use Corollary 4 (that generalizes the Jessen theorem) to define a measure $\lambda_{N, a}^{(k)}$ over $(E, \mathscr{B}(E))$, where $k \in \mathbf{N}$; consequently, we define also a theory of integration. In the case $I=\{1, \ldots, k\}$, the measure $\lambda_{N, a}^{(k)}$ coincides with the $k$-dimensional Lebesgue measure on $\mathbf{R}^{k}$.

In Section 3, we introduce the determinant of a class of infinite-dimensional matrices, called $(m, \sigma)$-standard, and we expose briefly a theory that generalizes the standard theory of the $m \times m$ matrices. Moreover, we prove that the determinant of a $(m, \sigma)$-standard matrix is equal to the product of its eigenvalues, as in the finite-dimensional case. In Section 4, we present the main result of our paper, that is, a change of variables formula for the integration of the biunique linear functions associated with the $(m, \sigma)$ standard matrices (Theorem 29). This result agrees with the analogous finite-dimensional result. In Section 5, we expose an application in the probabilistic framework, that is, the definition of the infinite-dimensional probability density of a random element. Moreover, we prove the formula of the density of such a random element composed with a $(m, \sigma)$-standard matrix. In Section 6, we expose some ideas for further study in the mathematical analysis and probability. 


\section{Construction of a Generalized Lebesgue Measure}

Suppose that $k \in \mathbf{N}, N \in \mathbf{R}^{+}$, and $I=\left\{n \in \mathbf{N}^{*}: n<M\right\}$, where $k+1 \leq M \leq+\infty$ and $a=\left(a_{n}: n \in I\right) \in\left(\mathbf{R}^{+}\right)^{I}$ such that there exists $\prod_{n \in I} a_{n} \in \mathbf{R}^{+}$. Moreover, indicate by $\mathscr{B}$, by $\mathscr{B}^{(k)}$, by Leb, and by Leb ${ }^{(k)}$, respectively, the Borel $\sigma$-algebra on $\mathbf{R}$, the Borel $\sigma$-algebra on $\mathbf{R}^{k}$, the Lebesgue measure on $\mathbf{R}$, and the Lebesgue measure on $\mathbf{R}^{k}$. Finally, for any topological space $E$ and for any $D \subset E$, indicate by $\mathscr{B}(D)$ the Borel $\sigma$ algebra on $D$.

Definition 1. Define the function $\|\cdot\|: \mathbf{R}^{I} \rightarrow[0,+\infty]$ by

$$
\|x\|=\sup _{n \in I}\left|x_{n}\right|, \quad \forall x=\left(x_{n}: n \in I\right) \in \mathbf{R}^{I},
$$

and define the following vector space on the field $\mathbf{R}$ :

$$
E=\left\{x \in \mathbf{R}^{I}:\|x\|<+\infty\right\} .
$$

Remark 2. E is a Banach space.

Proof. It is easy to prove that $\|\cdot\|$ is a norm on $E$; then, $E$ is a metric space with the distance $d: E \times E \rightarrow[0,+\infty)$ defined by $d(x, y)=\|x-y\|=\sup _{n \in I}\left|x_{n}-y_{n}\right|, \forall x=\left(x_{n}: n \in I\right) \in E$, and $\forall y=\left(y_{n}: n \in I\right) \in E$. Moreover, let $\left\{x_{i}\right\}_{i \in \mathbf{N}}$ be a Cauchy sequence on $E$; then, $\forall \varepsilon>0, \exists i_{0} \in \mathbf{N}$ such that $\forall i, j \in \mathbf{N}$ such that $i, j \geq i_{0}$, we have $\left\|x_{i}-x_{j}\right\|<\varepsilon$, and so, $\forall n \in I$, $\left|\left(x_{i}\right)_{n}-\left(x_{j}\right)_{n}\right|<\varepsilon$. Since $\mathbf{R}$ is complete, $\forall n \in I, \exists l_{n} \in \mathbf{R}$ such that $\lim _{j \rightarrow+\infty}\left(x_{j}\right)_{n}=l_{n}$; then, by setting $l=\left(l_{n}: n \in I\right) \in \mathbf{R}^{I}$, we have

$$
\begin{aligned}
\left|\left(x_{i}\right)_{n}-l_{n}\right| & =\lim _{j \rightarrow+\infty}\left|\left(x_{i}\right)_{n}-\left(x_{j}\right)_{n}\right| \leq \varepsilon \\
& \Longrightarrow\left\|x_{i}-l\right\|=\sup _{n \in I}\left|\left(x_{i}\right)_{n}-l_{n}\right| \leq \varepsilon .
\end{aligned}
$$

This implies that $l \in E$ and $\lim _{i \rightarrow+\infty} x_{i}=l$; then, $E$ is complete, and so it is a Banach space.

In order to develop the next arguments, for any set $I$ and for any $H \subset I$ define the projection $\pi_{H}$ on $\mathbf{R}^{H}$ as the function $\pi_{H}: \mathbf{R}^{I} \rightarrow \mathbf{R}^{H}$ given by $\pi_{H}\left(x_{n}: n \in I\right)=\left(x_{h}: h \in H\right)$. We will use the following result, whose proof can be found, for example, in Rao [6, page 346].

Theorem 3 (Jessen theorem). Let I be a set and, for any $i \in I$, let $\left(E_{i}, \mathscr{E}_{i}, \mu_{i}\right)$ be a probability space. Then, over the measurable space $\left(\prod_{i \in I} E_{i}, \bigotimes_{i \in I} \mathscr{E}_{i}\right)$, there is a unique probability measure $\mu$, indicated by $\bigotimes_{i \in I} \mu_{i}$, such that, for any $H \subset I$ such that $|H|<+\infty$ and for any $A=\prod_{h \in H} A_{h} \times \prod_{i \in I \backslash H} E_{i} \in \bigotimes_{i \in I} \mathscr{E}_{i}$, where $A_{h} \in \mathscr{E}_{h}, \forall h \in H$, we have $\mu(A)=\prod_{h \in H} \mu_{h}\left(A_{h}\right)$. In particular, if I is countable, then $\mu(A)=\prod_{i \in I} \mu_{i}\left(A_{i}\right)$ for any $A=\prod_{i \in I} A_{i} \in \bigotimes_{i \in I} \mathscr{E}_{i}$.

Corollary 4. Let I be a set and, for any $i \in I$, let $\left(E_{i}, \mathscr{E}_{i}, \mu_{i}\right)$ be a measure space such that $\mu_{i}$ is finite. Moreover, suppose that, for some countable set $J \subset I, \mu_{i}$ is a probability measure for any $i \in I \backslash J$ and $\prod_{j \in J} \mu_{j}\left(E_{j}\right) \in \mathbf{R}^{+}$. Then, over the measurable space $\left(\prod_{i \in I} E_{i}, \bigotimes_{i \in I} \mathscr{E}_{i}\right)$, there is a unique finite measure $\mu$, indicated by $\bigotimes_{i \in I} \mu_{i}$, such that, for any $H \subset I$ such that $|H|<+\infty$ and for any $A=\prod_{h \in H} A_{h} \times \prod_{i \in I \backslash H} E_{i} \in \bigotimes_{i \in I} \mathscr{E}_{i}$, where $A_{h} \in$ $\mathscr{E}_{h}, \forall h \in H$, one has $\mu(A)=\prod_{h \in H} \mu_{h}\left(A_{h}\right) \prod_{j \in J \backslash H} \mu_{j}\left(E_{j}\right)$. In particular, if $I$ is countable, then $\mu(A)=\prod_{i \in I} \mu_{i}\left(A_{i}\right)$ for any $A=\prod_{i \in I} A_{i} \in \bigotimes_{i \in I} \mathscr{E}_{i}$.

Proof. For any $i \in I, \overline{\mu_{i}}=\left(\mu_{i} / \mu_{i}\left(E_{i}\right)\right)$ is a probability measure; then, if $\bar{\mu}=\bigotimes_{i \in I} \overline{\mu_{i}}$ is the probability measure defined by Theorem 3, the finite measure $\mu=\left(\prod_{j \in J} \mu_{j}\left(E_{j}\right)\right) \bar{\mu}$ satisfies the statement.

Since for any $n \in I \backslash\{1, \ldots, k\}$ the measure $(1 / 2 N) \operatorname{Leb}\left(\cdot \cap\left[-N a_{n}, N a_{n}\right]\right)$ is a finite measure over $(\mathbf{R}, \mathscr{B})$, from Corollary 4 we can define the $\sigma$-finite measure $\lambda_{N, a}^{(k)}$ over $(E, \mathscr{B}(E))$ in the following manner:

$$
\lambda_{N, a}^{(k)}=\frac{1}{(2 N)^{k}} \operatorname{Leb}^{(k)} \otimes \bigotimes_{n \in I \backslash\{1, \ldots, k\}} \frac{1}{2 N} \operatorname{Leb}\left(\cdot \cap\left[-N a_{n}, N a_{n}\right]\right) .
$$

Remark 5. For any $N \in \mathbf{R}^{+}$, we have

$$
\lambda_{N, a}^{(k)}(E)= \begin{cases}\prod_{n \in I} a_{n} & \text { if } k=0 \\ +\infty & \text { if } k \in \mathbf{N}^{*}\end{cases}
$$

Proof. If $N \in \mathbf{R}^{+}$and $k=0$, from Corollary 4, we have

$$
\lambda_{N, a}^{(k)}(E)=\prod_{n \in I} \frac{1}{2 N} \operatorname{Leb}\left(\left[-N a_{n}, N a_{n}\right]\right)=\prod_{n \in I} a_{n} .
$$

Analogously, if $N \in \mathbf{R}^{+}$and $k \in \mathbf{N}^{*}$ :

$$
\lambda_{N, a}^{(k)}(E)=\frac{1}{(2 N)^{k}} \operatorname{Leb}^{(k)}\left(\mathbf{R}^{k}\right) \prod_{n \in I \backslash\{1, \ldots, k\}} a_{n}=+\infty .
$$

\section{Infinite-Dimensional Matrices}

Definition 6. Let $A=\left(a_{i j}\right)_{i, j \in I}$ be a real matrix $I \times$ $I$ (eventually infinite, if $I=\mathbf{N}^{*}$ ); then, define the linear function $A=\left(a_{i j}\right)_{i, j \in I}: E \rightarrow \mathbf{R}^{I}$, and write $x \rightarrow A x$, in the following manner:

$$
(A x)_{i}=\sum_{j \in I} a_{i j} x_{j}, \quad \forall x \in E, \forall i \in I,
$$

on condition that, for any $i \in I$, the sum in (8) converges to a real number.

Proposition 7. Let $A=\left(a_{i j}\right)_{i, j \in I}$ be a real matrix $I \times I$; then

(1) the linear function $A=\left(a_{i j}\right)_{i, j \in I}: E \rightarrow \mathbf{R}^{I}$ given by (8) is defined if and only if, for any $i \in I, \sum_{j \in I}\left|a_{i j}\right|<+\infty$;

(2) $A(E) \subset E$ and $A$ is continuous if and only if $\sup _{i \in I} \sum_{j \in I}\left|a_{i j}\right|<+\infty$; moreover, in this case, $\|A\|=$ $\sup _{i \in I} \sum_{j \in I}\left|a_{i j}\right|$. 
Proof. (1) Suppose that the function $A=\left(a_{i j}\right)_{i, j \in I}: E \rightarrow \mathbf{R}^{I}$ is defined; then, $\forall i \in I$; let $x=\left(x_{n}: n \in I\right) \in E$ be such that $x_{n}=1$ if $a_{i n} \geq 0$, and $x_{n}=-1$ if $a_{\text {in }}<0$; since $A x \in \mathbf{R}^{I}$, we have

$$
\sum_{j \in I}\left|a_{i j}\right|=(A x)_{i} \in \mathbf{R}
$$

Conversely, suppose that $\sum_{j \in I}\left|a_{i j}\right|<+\infty, \forall i \in I$; then, $\forall x \in$ $E$ and $\forall i \in I, \sum_{j \in I}\left(a_{i j} x_{j}\right)^{+} \leq \sum_{j \in I}\left|a_{i j}\right|\left|x_{j}\right| \leq \sum_{j \in I}\left|a_{i j}\right|\|x\|<$ $+\infty$; analogously, $\sum_{j \in I}\left(a_{i j} x_{j}\right)^{-}<+\infty$, from which $(A x)_{i}=$ $\sum_{j \in I}\left(a_{i j} x_{j}\right)^{+}-\sum_{j \in I}\left(a_{i j} x_{j}\right)^{-} \in \mathbf{R}$, and so $A x \in \mathbf{R}^{I}$.

(2) If $A(E) \subset E$ and $A$ is continuous, from the previous arguments, we have that, $\forall i \in I$, there exists $x \in E$ such that $\|x\|=1$ and such that

$$
\begin{aligned}
\sum_{j \in I}\left|a_{i j}\right| & =(A x)_{i} \leq\|A x\| \leq\|A\|<+\infty \\
& \Longrightarrow \sup _{i \in I} \sum_{j \in I}\left|a_{i j}\right| \leq\|A\|<+\infty .
\end{aligned}
$$

Conversely, if $\sup _{i \in I} \sum_{j \in I}\left|a_{i j}\right|<+\infty, \forall x \in E$, such that $\|x\|=$ 1 , we have

$$
\begin{aligned}
\|A x\| & =\sup _{i \in I}\left|(A x)_{i}\right|=\sup _{i \in I}\left|\sum_{j \in I} a_{i j} x_{j}\right| \leq \sup _{i \in I} \sum_{j \in I}\left|a_{i j}\right|<+\infty \\
& \Longrightarrow\|A\|=\sup _{x \in E:\|x\|=1}\|A x\| \leq \sup _{i \in I} \sum_{j \in I}\left|a_{i j}\right|<+\infty .
\end{aligned}
$$

Finally, if $\sup _{i \in I} \sum_{j \in I}\left|a_{i j}\right|<+\infty$, from (10) and (11) we have

$$
\|A\|=\sup _{i \in I} \sum_{j \in I}\left|a_{i j}\right| \text {. }
$$

Definition 8. A linear function $A=\left(a_{i j}\right)_{i, j \in I}: E \rightarrow E$ is called $(m, \sigma)$-standard, where $m \in I \cup\{0\}$ and $\sigma: I \backslash$ $\{1, \ldots, m\} \rightarrow I \backslash\{1, \ldots, m\}$ is an increasing function, if
(1) $a_{i j}=0, \forall(i, j) \notin(\{1, \ldots, m\} \times I) \cup$ $\bigcup_{n \in I \backslash\{1, \ldots, m\}}\{(n, \sigma(n))\} ;$

(2) there exists $\prod_{n \in I \backslash\{1, \ldots, m\}: \lambda_{n} \neq 0} \lambda_{n} \in \mathbf{R}^{*}$, where $\lambda_{n}=$ $a_{n, \sigma(n)}, \forall n \in I \backslash\{1, \ldots, m\}$.

Moreover, indicate by $A_{m}$ the matrix $\left(a_{i j}\right)_{i, j \in\{1, \ldots, m\}} \in$ $M_{m}(\mathbf{R})$. Finally, indicate by $\mathscr{M}_{(m, \sigma)}(E)$ the set of the linear $(m, \sigma)$-standard functions from $E$ to $E$.

Remark 9. Let $A=\left(a_{i j}\right)_{i, j \in I}: E \rightarrow E$ be a linear $(m, \sigma)$-standard function. Then, $A$ is continuous; moreover, $\sigma$ is biunique if and only if $\sigma(n)=n, \forall n \in I \backslash\{1, \ldots, m\}$.

Proof. From the point 1 of Definition 8,

$$
\sup _{i \in I} \sum_{j \in I}\left|a_{i j}\right|=\sup \left\{\sup _{i \in\{1, \ldots, m\}} \sum_{j \in I}\left|a_{i j}\right|, \sup _{n \in I \backslash\{1, \ldots, m\}: \lambda_{n} \neq 0}\left|\lambda_{n}\right|\right\} .
$$

We have $\sup _{i \in\{1, \ldots, m\}} \sum_{j \in I}\left|a_{i j}\right|<+\infty$ from Proposition 7; moreover, if $\lambda_{n}=0$ for $n$ sufficiently large, obviously $\sup _{n \in I \backslash\{1, \ldots, m\}: \lambda_{n} \neq 0}\left|\lambda_{n}\right|<+\infty$; otherwise, consider the subsequence $\left\{\lambda_{n_{k}}\right\}_{k \in \mathrm{N}}=\left\{\lambda_{n} \neq 0: n \in I \backslash\{1, \ldots, m\}\right\}$; from the point 2 of Definition 8 , we obtain $\lim _{k \rightarrow+\infty} \lambda_{n_{k}}=1$, and so $\sup _{n \in I \backslash\{1, \ldots, m\}: \lambda_{n} \neq 0}\left|\lambda_{n}\right|<+\infty$ again. Then, $\sup _{i \in I} \sum_{j \in I}\left|a_{i j}\right|<$ $+\infty$, from which $A$ is continuous from Proposition 7. Moreover, $\sigma$ is biunique if and only if $\sigma(n)=n, \forall n \in I \backslash\{1, \ldots, m\}$, because $\sigma$ is increasing.

Proposition 10. Let $A=\left(a_{i j}\right)_{i, j \in I}: E \rightarrow E$ be a linear $(m, \sigma)$-standard function; then, $A$ is biunique if and only if the matrix $A_{m}$ is invertible, $a_{n, \sigma(n)} \neq 0, \forall n \in I \backslash\{1, \ldots, m\}$, and $\sigma$ is biunique.

Proof. If $A_{m}$ is invertible and $a_{n, \sigma(n)} \neq 0, \forall n \in I \backslash\{1, \ldots, m\}$, let $x, y \in E$ be such that $A x=A y$; from the point 1 of Definition $8, \forall n \in I \backslash\{1, \ldots, m\}$, we have $a_{n, \sigma(n)} x_{\sigma(n)}=$ $a_{n, \sigma(n)} y_{\sigma(n)}$, from which $x_{\sigma(n)}=y_{\sigma(n)}$; then, if $\sigma$ is biunique, we have $\sigma(n)=n$, and so $\left(x_{n}: n>m\right)=\left(y_{n}: n>m\right)$. This implies that $A_{m}{ }^{t}\left(x_{1}, \ldots, x_{m}\right)=A_{m}{ }^{t}\left(y_{1}, \ldots, y_{m}\right)$, and so $\left(x_{1}, \ldots, x_{m}\right)=\left(y_{1}, \ldots, y_{m}\right)$; then, $x=y$; that is, $A$ is injective. Moreover, $\forall y \in E$, define $x \in E$ in the following manner:

$$
\begin{gathered}
x_{n}=\frac{y_{n}}{a_{n n}}, \quad \forall n \in I \backslash\{1, \ldots, m\}, \\
{ }^{t}\left(x_{1}, \ldots, x_{m}\right)=A_{m}^{-1}\left({ }^{t}\left(z_{1}, \ldots, z_{m}\right)\right),
\end{gathered}
$$

where

$$
z_{i}=y_{i}-\sum_{n>m} a_{i n} x_{n}, \quad \forall i \in\{1, \ldots, m\}
$$

It is easy to prove that $A x=y$; that is, $A$ is surjective.

Conversely, if $A$ is biunique, let $\mathbf{x}, \mathbf{y} \in \mathbf{R}^{m}$ be such that $A_{m} \mathbf{x}=A_{m} \mathbf{y}$, and let $\bar{x}, \bar{y} \in E$ be such that $\bar{x}_{n}=x_{n}, \bar{y}_{n}=y_{n}$, $\forall n \in\{1, \ldots, m\}$, and $\bar{x}_{n}=\bar{y}_{n}=0, \forall n \in I \backslash\{1, \ldots, m\}$. We have $A_{m} \mathbf{x}=\boldsymbol{\pi}_{\{1, \ldots, m\}}(A \bar{x}), A_{m} \mathbf{y}=\boldsymbol{\pi}_{\{1, \ldots, m\}}(A \bar{y})$, and $(A \bar{x})_{n}=$ $(A \bar{y})_{n}=0, \forall n \in I \backslash\{1, \ldots, m\}$, from which $A \bar{x}=A \bar{y}$; then, since $A$ is biunique, we have $\bar{x}=\bar{y}$, and so $\mathbf{x}=\mathbf{y}$. Then, the linear function $\mathbf{x} \rightarrow A_{m} \mathbf{x}$ is injective; that is, $A_{m}$ is invertible. Moreover, we have $a_{n, \sigma(n)} \neq 0, \forall n \in I \backslash\{1, \ldots, m\}$; in fact, by supposing by contradiction that $a_{\bar{n}, \sigma(\bar{n})}=0$, for some $\bar{n}>m$, then $A(E) \subset\left\{x \in E: x_{\bar{n}}=0\right\} \varsubsetneqq E$, and this should contradict the fact that $A$ is surjective. Moreover, $\sigma$ must be injective; in fact, by supposing that $\sigma\left(n_{1}\right)=\sigma\left(n_{2}\right)$, for some $m<n_{1}<$ $n_{2}$, then $A(E) \subset\left\{x \in E: x_{n_{1}} a_{n_{2}, \sigma\left(n_{2}\right)}=x_{n_{2}} a_{n_{1}, \sigma\left(n_{1}\right)}\right\} \varsubsetneqq$ $E$ (a contradiction). Finally, $\sigma$ must be surjective, because otherwise, $\forall y \in E$ and $\forall \bar{n} \in(I \backslash\{1, \ldots, m\}) \backslash \sigma(I \backslash\{1, \ldots, m\})$, we could choose arbitrarily $x_{\bar{n}} \in \mathbf{R}$ in order to determine $x=\left(x_{n}: n \in I\right) \in E$ such that $A x=y$. Then, $A$ should not be injective (again a contradiction).

In order to study the inverse of $A$, we must define the following concept, that generalizes the determinant of a $m \times m$ matrix (see, e.g., the theory in Lang's book [7]). 
Definition 11. Let $A=\left(a_{i j}\right)_{i, j \in I}: E \rightarrow E$ be a linear $(m, \sigma)$-standard function; define the determinant of $A$, and call it $\operatorname{det}_{(m, \sigma)} A$, or $\operatorname{det} A$, the real number:

$$
\operatorname{det}_{(m, \sigma)} A= \begin{cases}\operatorname{det} A_{m} \prod_{n \in I \backslash\{1, \ldots, m\}} \lambda_{n} & \text { if } \sigma \text { is biunique } \\ 0 & \text { if } \sigma \text { is not biunique. }\end{cases}
$$

Remark 12. If $A \in \mathscr{M}_{\left(m_{1}, \sigma_{1}\right)}(E) \cap \mathscr{M}_{\left(m_{2}, \sigma_{2}\right)}(E)$, then $\operatorname{det}_{\left(m_{1}, \sigma_{1}\right)} A=\operatorname{det}_{\left(m_{2}, \sigma_{2}\right)} A$.

Proof. Suppose that $m_{1} \leq m_{2}$; then, we have $\left.\sigma_{1}\right|_{I \backslash\left\{1, \ldots, m_{2}\right\}}=\sigma_{2}$. If $\sigma_{1}$ is biunique, $\sigma_{2}$ is biunique too, and $\sigma_{1}(n)=n, \forall n \in$ $\left\{m_{1}+1, \ldots, m_{2}\right\}$; then

$$
\begin{aligned}
\operatorname{det}_{\left(m_{1}, \sigma_{1}\right)} A & =\operatorname{det} A_{m_{1}} \prod_{n \in I \backslash\left\{1, \ldots, m_{1}\right\}} \lambda_{n} \\
& =\operatorname{det} A_{m_{1}} \prod_{p \in\left\{m_{1}+1, \ldots, m_{2}\right\}} \lambda_{p} \prod_{n \in I \backslash\left\{1, \ldots, m_{2}\right\}} \lambda_{n} \\
& =\operatorname{det} A_{m_{2}} \prod_{n \in I \backslash\left\{1, \ldots, m_{2}\right\}} \lambda_{n}=\operatorname{det}_{\left(m_{2}, \sigma_{2}\right)} A .
\end{aligned}
$$

Instead, if $\sigma_{1}$ is not biunique, then either $\sigma_{2}$ is not biunique, or $\sigma_{2}$ is biunique, but not $\left.\sigma_{1}\right|_{\left\{m_{1}+1, \ldots, m_{2}\right\}}$. In the first case, we have

$$
\operatorname{det}_{\left(m_{1}, \sigma_{1}\right)} A=0=\operatorname{det}_{\left(m_{2}, \sigma_{2}\right)} A \text {. }
$$

In the second case, we have $\operatorname{det} A_{m_{2}}=0$, and so

$$
\operatorname{det}_{\left(m_{1}, \sigma_{1}\right)} A=0=\operatorname{det} A_{m_{2}} \prod_{n \in I \backslash\left\{1, \ldots, m_{2}\right\}} \lambda_{n}=\operatorname{det}_{\left(m_{2}, \sigma_{2}\right)} A .
$$

Proposition 13. Let $A=\left(a_{i j}\right)_{i, j \in I}: E \rightarrow E$ be a linear $(m, \sigma)$ standard function, with $\sigma$ being biunique, let $s, t \in I, s<t$, let $p=\max \{t, m\}$, and let the function $\tau=\left.\sigma\right|_{I \backslash\{1, \ldots, p\}}$; then

(1) if there exist $u=\left(u_{n}: n \in I\right) \in E, v=\left(v_{n}: n \in I\right) \in E$, and $c_{1}, c_{2} \in \mathbf{R}$ such that $\sum_{n \in I}\left|u_{n}\right|<+\infty, \sum_{n \in I}\left|v_{n}\right|<$ $+\infty, a_{t j}=c_{1} u_{j}+c_{2} v_{j}, \forall j \in I$, by indicating by $U$ and $V$ the linear functions obtained by substituting the th row of $A$ for $u$ and $v$, respectively, then $U$ and $V$ are $(p, \tau)$-standard and $\operatorname{det} A=c_{1} \operatorname{det} U+c_{2} \operatorname{det} V$;

(2) if $B=\left(b_{i j}\right)_{i, j \in I}: E \rightarrow E$ is the linear function obtained by exchanging the sth row of $A$ for the th row of $A$, then $B$ is $(p, \tau)$-standard and $\operatorname{det} B=-\operatorname{det} A$;

(3) if $C=\left(c_{i j}\right)_{i, j \in I}: E \rightarrow E$ is the linear function obtained by substituting the th row of $A$ for the sth row of $A$, then $C$ is $(p, \tau)$-standard and $\operatorname{det} C=0$.

Proof. (1) Since $\sigma$ is biunique, we have $\sigma(n)=n, \forall n \in$ $I \backslash\{1, \ldots, m\}$, and so we can prove easily that $U$ and $V$ are $(p, \tau)$-standard; moreover, $\operatorname{det} A=\operatorname{det} A_{p} \prod_{n \in I \backslash\{1, \ldots, p\}} \lambda_{n}$ and $\operatorname{det} A_{p}=c_{1} \operatorname{det} U_{p}+c_{2} \operatorname{det} V_{p}$; then

$$
\begin{aligned}
\operatorname{det} A & =\left(c_{1} \operatorname{det} U_{p}+c_{2} \operatorname{det} V_{p}\right) \prod_{n \in I \backslash\{1, \ldots, p\}} \lambda_{n} \\
& =c_{1} \operatorname{det} U_{p} \prod_{n \in I \backslash\{1, \ldots, p\}} \lambda_{n}+c_{2} \operatorname{det} V_{p} \prod_{n \in I \backslash\{1, \ldots, p\}} \lambda_{n} \\
& =c_{1} \operatorname{det} U+c_{2} \operatorname{det} V .
\end{aligned}
$$

(2) As we observed in the proof of the point $1, B$ is $(p, \tau)$ standard; moreover, $\operatorname{det} B=\operatorname{det} B_{p} \prod_{n \in I \backslash\{1, \ldots, p\}} \lambda_{n}$, where $B_{p}$ is the matrix obtained by exchanging the sth row of $A_{p}$ for the $t$ th row of $A_{p}$; then, $\operatorname{det} B_{p}=-\operatorname{det} A_{p}$, from which

$$
\operatorname{det} B=-\operatorname{det} A_{p} \prod_{n \in I \backslash\{1, \ldots, p\}} \lambda_{n}=-\operatorname{det} A .
$$

(3) Since the sth row of $C$ and the $t$ th row of $C$ are equal, by exchanging these rows among themselves we obtain again the matrix $C$; then, from the point 2 , we have $\operatorname{det} C=-\operatorname{det} C$, from which $\operatorname{det} C=0$.

Remark 14. Let $A=\left(a_{i j}\right)_{i, j \in I}: E \rightarrow E$ be a linear $(m, \sigma)$-standard function; then, $A$ is biunique if and only if $\operatorname{det} A \neq 0$.

Proof. If $A$ is biunique, from Proposition $10 \sigma$ is biunique, and so $\operatorname{det} A=\operatorname{det} A_{m} \prod_{n \in I \backslash\{1, \ldots, m\}} \lambda_{n} ;$ moreover, we have $\operatorname{det} A_{m} \neq 0$ and $\lambda_{n} \neq 0, \forall n \in I \backslash\{1, \ldots, m\}$, from which $\prod_{n \in I \backslash\{1, \ldots, m\}} \lambda_{n}=\prod_{n \in I \backslash\{1, \ldots, m\}: \lambda_{n} \neq 0} \lambda_{n} \neq 0$; then, $\operatorname{det} A \neq 0$.

Conversely, if $\operatorname{det} A \neq 0$, then $\sigma$ is biunique by definition of $\operatorname{det} A$, and so $0 \neq \operatorname{det} A=\operatorname{det} A_{m} \prod_{n \in I \backslash\{1, \ldots, m\}} \lambda_{n}$; this implies that $\operatorname{det} A_{m} \neq 0$ and $\lambda_{n} \neq 0, \forall n \in I \backslash\{1, \ldots, m\}$; then, from Proposition 10, $A$ is biunique.

Definition 15. Let $A=\left(a_{i j}\right)_{i, j \in I}: E \rightarrow E$ be a linear $(m, \sigma)$ standard function; define the $I \times I$ matrix $\operatorname{cof} A=\left(A_{i j}\right)_{i, j \in I}$ by

$$
A_{i j}=(-1)^{i+j} \operatorname{det}(A(1 \cdots \widehat{i} \cdots \mid 1 \cdots \widehat{j} \cdots)),
$$

where $A(1 \cdots \hat{i} \cdots \mid 1 \cdots \hat{j} \cdots)$ is the $(I \backslash\{i\}) \times(I \backslash\{j\})$ matrix obtained by deleting the $i$ th row and the $j$ th column of $A$.

Proposition 16. Let $A=\left(a_{i j}\right)_{i, j \in I}: E \rightarrow E$ be a linear $(m, \sigma)$-standard function; then, for any $i \in I$, one has

$$
\operatorname{det} A=\sum_{j \in I} a_{i j} A_{i j} \text {. }
$$

Proof. Suppose that $\sigma$ is biunique; then, $\forall i \in\{1, \ldots, m\}$, we have

$$
\begin{aligned}
\operatorname{det} A=\operatorname{det} A_{m} \prod_{n \in I \backslash\{1, \ldots, m\}} \lambda_{n} & =\sum_{j=1}^{m} a_{i j}\left(A_{m}\right)_{i j}\left(\prod_{n \in I \backslash\{1, \ldots, m\}} \lambda_{n}\right) \\
& =\sum_{j=1}^{m} a_{i j} A_{i j} .
\end{aligned}
$$


Moreover, $\forall i \in\{1, \ldots, m\}$ and $\forall j>m$, the matrix $A(1 \cdots \widehat{i} \cdots \mid 1 \cdots \widehat{j} \cdots)$ is $(m-1, \bar{\sigma})$-standard, where

$$
\bar{\sigma}: I \backslash\{1, \ldots, m-1\} \longrightarrow I \backslash\{1, \ldots, m-1\}
$$

is not surjective because $m \notin \bar{\sigma}(I \backslash\{1, \ldots, m-1\})$, and so $A_{i j}=0$; then, $\operatorname{det} A=\sum_{j \in I} a_{i j} A_{i j}$. Finally, $\forall i>m$, we have $a_{i j}=0, \forall j \neq i$; then

$$
\begin{aligned}
\sum_{j \in I} a_{i j} A_{i j} & =a_{i i} A_{i i} \\
& =a_{i i}(-1)^{2 i} \operatorname{det} A_{m} \prod_{n \in I \backslash\{1, \ldots, m, i\}} \lambda_{n} \\
& =\operatorname{det} A_{m} \prod_{n \in I \backslash\{1, \ldots, m\}} \lambda_{n}=\operatorname{det} A .
\end{aligned}
$$

Instead, if $\sigma$ is not biunique, $\forall i, j \in\{1, \ldots, m\}$, the matrix $A(1 \cdots \hat{i} \cdots \mid 1 \cdots \widehat{j} \cdots)$ is $(m-1, \widehat{\sigma})$-standard, where $\widehat{\sigma}(n)=$ $\sigma(n+1), \forall n>m-1$; then, $\widehat{\sigma}$ is not biunique, from which $A_{i j}=0$. Moreover, $\forall i \in\{1, \ldots, m\}$ and $\forall j>m$, as in the case $\sigma$ being biunique, we have $A_{i j}=0$. Finally, $\forall i>m$, we have $a_{i j}=0, \forall j \neq \sigma(i)$; then

$$
\sum_{j \in I} a_{i j} A_{i j}=a_{i, \sigma(i)} A_{i, \sigma(i)}
$$

Moreover, the matrix $A(1 \ldots \hat{i} \ldots \mid 1 \ldots \widehat{\sigma(i)} \ldots)$ is $(m, \widetilde{\sigma})$ standard, where the function $\widetilde{\sigma}: I \backslash\{1, \ldots, m, i\} \rightarrow I \backslash$ $\{1, \ldots, m, \sigma(i)\}$ is not biunique; in fact, in this case necessarily $\sigma(i)=i$, and so $\sigma$ should be biunique (a contradiction); then, we have $A_{i, \sigma(i)}=0$, from which

$$
\operatorname{det} A=0=\sum_{j \in I} a_{i j} A_{i j}
$$

Corollary 17. Let $A=\left(a_{i j}\right)_{i, j \in I}: E \rightarrow E$ be a biunique and linear $(m, \sigma)$-standard function; then, $A^{-1}: E \rightarrow E$ is a linear $(m, \sigma)$-standard function $A^{-1}=\left(b_{i j}\right)_{i, j \in I}$; moreover

$$
A^{-1}=\frac{1}{\operatorname{det} A}^{t}(\operatorname{cof} A) \text {. }
$$

Proof. From Proposition 16, we have

$$
\sum_{n \in I} a_{i n} A_{i n}=\operatorname{det} A
$$

Moreover, we have

$$
\sum_{n \in I} a_{i n} A_{j n}=0, \quad \forall i, j \in I, i \neq j
$$

in fact, from Proposition 16, the left side of (31) is equal to $\operatorname{det} C$, where $C$ is the $(p, \tau)$-standard matrix obtained by substituting the $i$ th row of $A$ for the $j$ th row of $A$; then, from Proposition 13, we have $\operatorname{det} C=0$. This implies that

$$
\sum_{n \in I} a_{i n} A_{j n}=(\operatorname{det} A) \delta_{i j}, \quad \forall i, j \in I,
$$

where $\delta_{i j}$ is the Kronecker symbol, and so

$$
\left(A^{t}(\operatorname{cof} A)\right)_{i j}=(\operatorname{det} A) \delta_{i j}, \quad \forall i, j \in I,
$$

from which the formula (29) follows. Moreover, as we observed in the proof of Proposition $16, \forall i \in\{1, \ldots, m\}$ and $\forall j>m$, we have $A_{i j}=0$; finally, $\forall i, j>m$ such that $i \neq j$, the matrix $A(1 \cdots \hat{i} \cdots \mid 1 \cdots \hat{j} \cdots)$ is $(m, \bar{\sigma})$-standard, where $\bar{\sigma}: I \backslash\{1, \ldots, m, i\} \rightarrow I \backslash\{1, \ldots, m, j\}$ is not surjective because $i \notin \bar{\sigma}(I \backslash\{1, \ldots, m, i\})$, and so $A_{i j}=0$ again; from formula (29), this implies that $A^{-1}$ is $(m, \sigma)$-standard.

Definition 18. Define the function $\|\cdot\|: \mathbf{C}^{I} \rightarrow[0,+\infty]$ by

$$
\|x\|=\sup _{n \in I}\left|x_{n}\right|, \quad \forall x=\left(x_{n}: n \in I\right) \in \mathbf{C}^{I} \text {, }
$$

and define the following vector space on the field $\mathbf{C}$, with the norm $\|\cdot\|$ :

$$
F=\left\{x \in \mathbf{C}^{I}:\|x\|<+\infty\right\} \supset E
$$

Definition 19. Let $A=\left(a_{i j}\right)_{i, j \in I}$ be a real matrix $I \times I$; then, define the linear function $A=\left(a_{i j}\right)_{i, j \in I}: F \rightarrow \mathrm{C}^{I}$ and write $x \rightarrow A x$, in the following manner:

$$
(A x)_{i}=\sum_{j \in I} a_{i j} x_{j}, \quad \forall x \in F, \forall i \in I
$$

on condition that, for any $i \in I$, the sum in (36) converges to a complex number.

Proposition 20. Let $A=\left(a_{i j}\right)_{i, j \in I}$ be a real matrix $I \times I$; then

(1) the linear function $A=\left(a_{i j}\right)_{i, j \in I}: F \rightarrow C^{I}$ given by (36) is defined if and only if, for any $i \in I, \sum_{j \in I}\left|a_{i j}\right|<$ $+\infty$.

(2) $A(F) \subset F$ and $A$ is continuous if and only if $\sup _{i \in I} \sum_{j \in I}\left|a_{i j}\right|<+\infty$; moreover, in this case $\|A\|=$ $\sup _{i \in I} \sum_{j \in I}\left|a_{i j}\right|$.

Proof. The proof is analogous to that one of Proposition 7.

Definition 21. Let $V$ be a vector space on $\mathbf{C}$, and let $T$ : $V \rightarrow V$ be a linear function; indicate by $V P(T)$ the set of the eigenvalues of $T$.

Proposition 22. Let $A=\left(a_{i j}\right)_{i, j \in I}: E \rightarrow$ E be a linear $(m, \sigma)$ standard function, with $\sigma$ biunique; then, by considering $A$ as a function from $F$ to $F$, one has

$$
V P(A)=V P\left(A_{m}\right) \cup\left\{\lambda_{n}: n \in I \backslash\{1, \ldots, m\}\right\} .
$$

Moreover

$$
\operatorname{det} A=\prod_{\lambda \in V P(A)} \lambda
$$


Proof. Let $\lambda \in \mathbf{C}$ be an eigenvalue of $A_{m}$, let $\mathbf{x} \in \mathbf{C}^{m} \backslash\{\mathbf{0}\}$ be the corresponding eigenvector, and let $y \in \mathbf{C}^{I} \backslash\{0\}$ be such that $y_{n}=x_{n}, \forall n \in\{1, \ldots, m\}$, and $y_{n}=0, \forall n \in I \backslash\{1, \ldots, m\}$. We have $(A y)_{n}=(A x)_{n}=(\lambda x)_{n}=(\lambda y)_{n}, \forall n \in\{1, \ldots, m\}$, and $(A y)_{n}=0=(\lambda y)_{n}, \forall n \in I \backslash\{1, \ldots, m\}$, from which $A y=\lambda y$, and so $\lambda \in V P(A)$. Moreover, $\forall n \in I \backslash\{1, \ldots, m\}$, since $\sigma$ is biunique, from the Remark 9, we have $\sigma(n)=n$. If $a_{i n}=0, \forall i \in\{1, \ldots, m\}$, let $x \in \mathbf{R}^{I} \backslash\{0\}$ be such that $x_{i}=\delta_{i n}$, $\forall i \in I$; we have $A x=\lambda_{n} x$, and so $\lambda_{n} \in V P(A)$. Otherwise, suppose that $a_{i n} \neq 0$ for some $i \in\{1, \ldots, m\}$; if $\lambda_{n} \in V P\left(A_{m}\right)$, then $\lambda_{n} \in V P(A)$ by the previous arguments; conversely, if $\left(A_{m}-\lambda_{n} I_{m}\right) \mathbf{x} \neq \mathbf{0}, \forall \mathbf{x} \in \mathbf{C}^{m} \backslash\{\mathbf{0}\}$, the matrix $\left(A_{m}-\lambda_{n} I_{m}\right)$ is invertible and so there exists $\mathbf{x} \in \mathbf{R}^{m} \backslash\{\mathbf{0}\}$ such that $A_{m} \mathbf{x}-$ $\lambda_{n} \mathbf{x}={ }^{t}\left(-a_{1 n}, \ldots,-a_{i n}, \ldots,-a_{m n}\right)$; then, by considering $y \in$ $\mathbf{R}^{I} \backslash\{0\}$ such that $y_{i}=x_{i}, \forall i \in\{1, \ldots, m\}, y_{i}=\delta_{i n}, \forall i \in$ $I \backslash\{1, \ldots, m\}$, we have $A y=\lambda_{n} y$, and so $\lambda_{n} \in V P(A)$. Then

$$
V P\left(A_{m}\right) \cup\left\{\lambda_{n}: n \in I \backslash\{1, \ldots, m\}\right\} \subset V P(A) .
$$

Conversely, if $\lambda \in V P(A)$, we have $A x=\lambda x$, for some $x \in \mathbf{C}^{I} \backslash\{0\}$, and so, $\forall n \in I \backslash\{1, \ldots, m\}, \lambda_{n} x_{n}=(A x)_{n}=\lambda x_{n}$; then, by supposing $\lambda \notin\left\{\lambda_{n}: n \in I \backslash\{1, \ldots, m\}\right\}$, we have $x_{n}=0$, from which $x_{n} \neq 0$ for some $n \in\{1, \ldots, m\}$. Moreover, we have

$$
A_{m}{ }^{t}\left(x_{1}, \ldots, x_{m}\right)={ }^{t}\left((A x)_{1}, \ldots,(A x)_{m}\right)=\lambda^{t}\left(x_{1}, \ldots, x_{m}\right),
$$

and so $\lambda \in V P\left(A_{m}\right)$. Then, we have

$$
V P(A) \subset V P\left(A_{m}\right) \cup\left\{\lambda_{n}: n \in I \backslash\{1, \ldots, m\}\right\},
$$

from which (37) follows. Moreover, since $\sigma$ is biunique, from (37), we have

$$
\operatorname{det} A=\operatorname{det} A_{m} \prod_{n \in I \backslash\{1, \ldots, m\}} \lambda_{n}=\prod_{\lambda \in V P(A)} \lambda .
$$

\section{Change of Variables' Formula}

Definition 23. Let $k \in \mathbf{N}$, let $M, N \in \mathbf{R}^{+}$, and let $a=\left(a_{n}: n \in\right.$ I) $\in\left(\mathbf{R}^{+}\right)^{I}$ such that $\prod_{n \in I} a_{n} \in \mathbf{R}^{+}$; define the following sets in $\mathscr{B}(E)$ :

$$
\begin{aligned}
E_{N, a}^{(k)} & =\mathbf{R}^{k} \times \prod_{n \in I \backslash\{1, \ldots, k\}}\left[-N a_{n}, N a_{n}\right] ; \\
E_{M, N, a}^{(k)} & =[-M, M]^{k} \times \prod_{n \in I \backslash\{1, \ldots, k\}}\left[-N a_{n}, N a_{n}\right] .
\end{aligned}
$$

Definition 24. Let $a=\left(a_{n}: n \in I\right) \in\left(\mathbf{R}^{+}\right)^{I}$ and $b=\left(b_{n}: n \in\right.$ I) $\in\left(\mathbf{R}^{+}\right)^{I}$ be such that $\prod_{n \in I} a_{n} \in \mathbf{R}^{+}, \prod_{n \in I} b_{n} \in \mathbf{R}^{+}$; define $a b \in\left(\mathbf{R}^{+}\right)^{I}$ in the following manner:

$$
a b=\left(a_{n} b_{n}: n \in I\right) .
$$

Proposition 25. Let $A=\left(a_{i j}\right)_{i, j \in I}: E \rightarrow E$ be a biunique and linear $(m, \sigma)$-standard function; then, for any $a=\left(a_{n}\right.$ : $n \in I) \in\left(\mathbf{R}^{+}\right)^{I}$ such that $\prod_{n \in I} a_{n} \in \mathbf{R}^{+}$, there exists $b=\left(b_{n}\right.$ : $n \in I) \in\left(\mathbf{R}^{+}\right)^{I}$ such that $\prod_{n \in I} b_{n} \in \mathbf{R}^{+}$and such that, for any $k \in \mathbf{N}, k \geq m$, and for any $N \in \mathbf{R}^{+}$, one has

$$
A^{-1}\left(E_{N, a}^{(k)}\right)=E_{N, b}^{(k)}
$$

Proof. From Corollary 17, $A^{-1}=\left(b_{i j}\right)_{i, j \in I}: E \rightarrow E$ is a linear $(m, \sigma)$-standard function. By setting $\rho_{n}=b_{n n}, \forall n>m$, from (29), we have

$$
\begin{aligned}
\rho_{n} & =\frac{1}{a_{n n}}=\frac{1}{\lambda_{n}} \\
& \Longrightarrow \prod_{n \in I \backslash\{1, \ldots, m\}} \rho_{n}=\prod_{n \in I \backslash\{1, \ldots, m\}} \frac{1}{\lambda_{n}} \in \mathbf{R}^{*} .
\end{aligned}
$$

Set $b=\left(b_{n}: n \in I\right) \in\left(\mathbf{R}^{+}\right)^{I}$ such that

$$
\begin{gathered}
b_{n}=1, \quad \forall n \in\{1, \ldots, m\}, \\
\left(b_{n}: n>m\right)=\left(a_{n}: n>m\right)\left(\left|\rho_{n}\right|: n>m\right) .
\end{gathered}
$$

By definition of $b$, we have

$$
\prod_{n \in I} b_{n}=\left(\prod_{n \in I \backslash\{1, \ldots, m\}} a_{n}\right)\left(\prod_{n \in I \backslash\{1, \ldots, m\}} \frac{1}{\left|\lambda_{n}\right|}\right) \in \mathbf{R}^{+}
$$

moreover, for any $k \in \mathbf{N}, k \geq m$, and for any $N \in \mathbf{R}^{+}$, we have $A^{-1}\left(E_{N, a}^{(k)}\right) \subset E_{N, b}^{(k)}$. Analogously, it is possible to prove that $A\left(E_{N, b}^{(k)}\right) \subset E_{N, c}^{(k)}$, where

$$
\left(c_{n}: n>m\right)=\left(b_{n}: n>m\right)\left(\left|\lambda_{n}\right|: n>m\right)=\left(a_{n}: n>m\right) .
$$

Moreover, since $k \geq m$, we have $E_{N, c}^{(k)}=E_{N, a}^{(k)}$, and so $E_{N, b}^{(k)} \subset$ $A^{-1}\left(E_{N, a}^{(k)}\right)$, from which (45) follows.

Lemma 26. Let $A=\left(a_{i j}\right)_{i, j \in I}: E \rightarrow E$ be a biunique and linear $(m, \sigma)$-standard function; then, for any $M_{1} \in \mathbf{R}^{+}$and for any $a=\left(a_{n}: n \in I\right) \in\left(\mathbf{R}^{+}\right)^{I}$ such that $\prod_{n \in I} a_{n} \in \mathbf{R}^{+}$, there exist $M_{2}, M_{3} \in \mathbf{R}^{+}$and $b=\left(b_{n}: n \in I\right) \in\left(\mathbf{R}^{+}\right)^{I}, c=\left(c_{n}: n \in\right.$ $I) \in\left(\mathbf{R}^{+}\right)^{I}$ such that $\prod_{n \in I} b_{n} \in \mathbf{R}^{+}, \prod_{n \in I} c_{n} \in \mathbf{R}^{+}$, and such that, for any $k \in \mathbf{N}$ and for any $N \in \mathbf{R}^{+}$, one has

$$
\begin{gathered}
A^{-1}\left(E_{M_{1}, N, a}^{(k)}\right) \subset E_{M_{2}, N, b}^{(k)} ; \\
A\left(E_{M_{2}, N, b}^{(k)}\right) \subset E_{M_{3}, N, c}^{(k)} .
\end{gathered}
$$

Moreover, $\left(c_{n}: n>m\right)=\left(a_{n}: n>m\right)$.

Proof. From the Banach theorem of the open function (see also the exercise 5.14 in [8]), $A^{-1}$ is continuous; then, $\forall N \in$ $\mathbf{R}^{+}$and $\forall x \in E_{M_{1}, N, a}^{(k)}$, we have

$$
\left\|A^{-1}(x)\right\| \leq\left\|A^{-1}\right\|\|x\| \leq\left\|A^{-1}\right\| \max \left\{M_{1}, N,\|a\|\right\} .
$$


Set $M_{2}=\left\|A^{-1}\right\| \max \left\{M_{1}, N,\|a\|\right\}$ and $b=\left(b_{n}: n \in I\right) \in\left(\mathbf{R}^{+}\right)^{I}$ such that

$$
\begin{gathered}
b_{n}=\frac{M_{2}}{N}, \quad \forall n \in\{1, \ldots, m\}, \\
\left(b_{n}: n>m\right)=\left(a_{n}: n>m\right)\left(\left|\rho_{n}\right|: n>m\right),
\end{gathered}
$$

where $\rho_{n}, \forall n \in I$, is defined as in the proof of Proposition 25. By definition of $b$, we have

$$
\prod_{n \in I} b_{n}=\left(\frac{M_{2}}{N}\right)^{m}\left(\prod_{n \in I \backslash\{1, \ldots, m\}} a_{n}\right)\left(\prod_{n \in I \backslash\{1, \ldots, m\}} \frac{1}{\left|\lambda_{n}\right|}\right) \in \mathbf{R}^{+},
$$

and (50) holds. Analogously, it is possible to prove (51); moreover

$$
\left(c_{n}: n>m\right)=\left(b_{n}: n>m\right)\left(\left|\lambda_{n}\right|: n>m\right)=\left(a_{n}: n>m\right) .
$$

Remark 27. Let $A=\left(a_{i j}\right)_{i, j \in I}: E \rightarrow E$ be a linear $(m, \sigma)$ standard function; then, $A$ is $\mathscr{B}(E) / \mathscr{B}(E)$-measurable.

Proof. Let $\tau$ be the topology induced by the norm $\|\cdot\|$ on $E$; then, since $A$ is continuous by Remark $9, \forall B \in \tau$ we have $A^{-1}(B) \in \tau \subset \mathscr{B}(E)$. Moreover, since $\sigma(\tau)=\mathscr{B}(E)$, we have $A^{-1}(B) \in \mathscr{B}(E), \forall B \in \mathscr{B}(E)$.

Proposition 28. Let $\mu_{1}$ and $\mu_{2}$ be two measures on a measurable space $(S, \Sigma)$ that coincide on a $\pi$-system $\mathscr{I}$ on $S$; then, if $\sigma(\mathscr{I})=\Sigma$ and $\mu_{1}(S)=\mu_{2}(S)<+\infty$, then $\mu_{1}$ and $\mu_{2}$ coincide on $\sum$.

Proof. See, for example, Theorem 3.3 in Billingsley [9].

Now, we can prove the main result of our paper, that generalizes the change of variables formula for the integration of a biunique linear function on $\mathbf{R}^{m}$ with values in $\mathbf{R}^{m}$ (see, e.g., Lang's book [10]).

Theorem 29 (change of variables' formula). Let $A=$ $\left(a_{i j}\right)_{i, j \in I}: E \rightarrow E$ be a biunique and linear $(m, \sigma)$-standard function, let $a=\left(a_{n}: n \in I\right) \in\left(\mathbf{R}^{+}\right)^{I}$ be such that $\prod_{n \in I} a_{n} \in$ $\mathbf{R}^{+}$, and let $b \in\left(\mathbf{R}^{+}\right)^{I}$ be the sequence defined by Proposition 25 . Then, for any $k \in \mathbf{N}, k \geq m$, for any $N \in \mathbf{R}^{+}$, for any $B \in \mathscr{B}(E)$, and for any measurable function $f:(E, \mathscr{B}(E)) \rightarrow(\mathbf{R}, \mathscr{B})$ such that $f^{+}\left(\right.$or $\left.f^{-}\right)$is $\lambda_{N, a}^{(k)}$-integrable, one has

$$
\int_{B} f d \lambda_{N, a}^{(k)}=\int_{A^{-1}(B)} f(A)|\operatorname{det} A| d \lambda_{N, b}^{(k)} .
$$

Proof. $\forall n \in \mathbf{N}$, let $h_{n}: E \rightarrow E$ be the biunique and linear $(m, \sigma)$-standard function given by

$$
\begin{aligned}
& \left(h_{n}(x)\right)_{i}=\left(A_{n}\left(\pi_{\{1, \ldots, n\}}(x)\right)\right)_{i}, \quad \forall x \in E, \forall i \in\{1, \ldots, n\} ; \\
& \left(h_{n}(x)\right)_{i}=\lambda_{i} x_{i}, \quad \forall x \in E, \forall i \in I \backslash\{1, \ldots, n\} .
\end{aligned}
$$

Moreover, $\forall M_{1} \in \mathbf{R}^{+}$and $\forall a=\left(a_{n}: n \in I\right) \in\left(\mathbf{R}^{+}\right)^{I}$ such that $\prod_{n \in I} a_{n} \in \mathbf{R}^{+}$, let $M_{2}(n), M_{3}(n)$ be the constants, and let $b(n), c(n)$ be the sequences defined by Lemma 26 and the function $h_{n}$; finally, consider the analogous constants $M_{2}, M_{3}$, and the sequences $b, c$ defined by $A$. Observe that $M_{2}(n) \leq$ $M_{2},(b(n))_{i} \leq b_{i}, \forall i \in I, \forall n \in \mathbf{N}$. Suppose that $n \geq k \geq m$ and $N \in \mathbf{R}^{+}$; then, $\forall B=\prod_{p \in I} B_{p}$, where $B_{p} \in \mathscr{B}\left(\left[-M_{1}, M_{1}\right]\right)$, $\forall p \in\{1, \ldots, k\}, B_{p} \in \mathscr{B}\left(\left[-N a_{p}, N a_{p}\right]\right), \forall p>k$, we have

$$
\begin{aligned}
& \int_{B} d \lambda_{N, a}^{(k)} \\
& =\int_{\left(B_{1} \times \cdots \times B_{k}\right) \times \prod_{q>k} B_{q}} d\left(\left(\bigotimes_{p=1}^{k} \frac{1}{2 N} \operatorname{Leb}\right) \otimes\left(\left.\bigotimes_{q>k} \frac{1}{2 N} \operatorname{Leb}\right|_{\mathscr{B}\left(\left[-N a_{q}, N a_{q}\right]\right)}\right)\right) \\
& =\int_{\left(B_{1} \times \cdots \times B_{n}\right) \times \prod_{q>n} B_{q}} d\left(\left(\bigotimes_{p=1}^{n} \frac{1}{2 N} \operatorname{Leb}\right) \otimes\left(\left.\bigotimes_{q>n} \frac{1}{2 N} \operatorname{Leb}\right|_{\mathscr{B}\left(\left[-N a_{q}, N a_{q}\right]\right)}\right)\right) \\
& =\int_{B_{1} \times \cdots \times B_{n}} d\left(\bigotimes_{p=1}^{n} \frac{1}{2 N} \text { Leb }\right) \\
& \times \int_{\prod_{q>n} B_{q}} d\left(\left.\bigotimes_{q>n} \frac{1}{2 N} \operatorname{Leb}\right|_{\mathscr{B}\left(\left[-N a_{q}, N a_{q}\right]\right)}\right) \\
& =\int_{A_{n}^{-1}\left(B_{1} \times \cdots \times B_{n}\right)}\left|\operatorname{det} A_{n}\right| d\left(\bigotimes_{p=1}^{n} \frac{1}{2 N} \text { Leb }\right) \\
& \times \int_{\prod_{q>n^{1 / \lambda_{q}} B_{q}}} \prod_{q>n}\left|\lambda_{q}\right| d\left(\left.\bigotimes_{q>n} \frac{1}{2 N} \operatorname{Leb}\right|_{\mathscr{B}\left(\left[-N b_{q}, N b_{q}\right]\right)}\right) \\
& =\int_{h_{n}^{-1}(B)}\left|\operatorname{det} h_{n}\right| d\left(\left(\bigotimes_{p=1}^{n} \frac{1}{2 N} \operatorname{Leb}\right) \otimes\left(\left.\bigotimes_{q>n} \frac{1}{2 N} \operatorname{Leb}\right|_{\mathscr{B}\left(\left[-N b_{q}, N b_{q}\right]\right)}\right)\right) \\
& =\int_{h_{n}^{-1}(B)}\left|\operatorname{det} h_{n}\right| d\left(\left(\bigotimes_{p=1}^{k} \frac{1}{2 N} \operatorname{Leb}\right) \otimes\left(\left.\bigotimes_{q>k} \frac{1}{2 N} \operatorname{Leb}\right|_{\mathscr{B}\left(\left[-N b_{q}, N b_{q}\right]\right)}\right)\right) \\
& =\int_{h_{n}^{-1}(B)}\left|\operatorname{det} h_{n}\right| d \lambda_{N, b}^{(k)} \text {. }
\end{aligned}
$$

Consider the measures $\mu_{1}$ and $\mu_{2}$ on $\mathscr{B}\left(E_{M_{1}, N, a}^{(k)}\right)$ defined by

$$
\begin{aligned}
& \mu_{1}(B)=\int_{B} d \lambda_{N, a}^{(k)} ; \\
& \mu_{2}(B)=\int_{h_{n}^{-1}(B)}\left|\operatorname{det} h_{n}\right| d \lambda_{N, b}^{(k)} .
\end{aligned}
$$

From (58), $\mu_{1}$ and $\mu_{2}$ coincide on the set $\mathscr{I}=\{B \in$ $\left.\mathscr{B}\left(E_{M_{1}, N, a}^{(k)}\right): B=\prod_{p \in I} B_{p}\right\}$; since $\mathscr{I}$ is a $\pi$-system on $E_{M_{1}, N, a}^{(k)}$ such that $\sigma(\mathscr{I})=\mathscr{B}\left(E_{M_{1}, N, a}^{(k)}\right)$ and since $\mu_{1}\left(E_{M_{1}, N, a}^{(k)}\right)=$ $\mu_{2}\left(E_{M_{1}, N, a}^{(k)}\right)=\left(M_{1} / N\right)^{k} \prod_{p>k} a_{p}<+\infty$, from Proposition 28, we have that $\forall B \in \mathscr{B}\left(E_{M_{1}, N, a}^{(k)}\right)$ :

$$
\int_{E_{M_{1}, N, a}^{(k)}} 1_{B} d \lambda_{N, a}^{(k)}=\int_{E_{M_{2}, N, b}^{(k)}} 1_{B}\left(h_{n}\right)\left|\operatorname{det} h_{n}\right| d \lambda_{N, b}^{(k)} .
$$


This implies that if $\varphi:\left(E_{M_{3}, N, a}^{(k)}, \mathscr{B}\left(E_{M_{3}, N, a}^{(k)}\right)\right) \rightarrow([0,+\infty)$, $\mathscr{B}([0,+\infty)))$ is a simple function such that $\varphi(x)=0, \forall x \notin$ $E_{M_{1}, N, a}^{(k)}$, we have

$$
\int_{E_{M_{1}, N, a}^{(k)}} \varphi d \lambda_{N, a}^{(k)}=\int_{E_{M_{2}, N, b}^{(k)}} \varphi\left(h_{n}\right)\left|\operatorname{det} h_{n}\right| d \lambda_{N, b}^{(k)} .
$$

Then, if $l:\left(E_{M_{3}, N, a}^{(k)}, \mathscr{B}\left(E_{M_{3}, N, a}^{(k)}\right)\right) \rightarrow([0,+\infty), \mathscr{B}([0,+\infty)))$ is a measurable function such that $\varphi(x)=0, \forall x \notin$ $E_{M_{1}, N, a}^{(k)}$, and $\left\{\varphi_{i}\right\}_{i \in \mathbf{N}}$ is a sequence of increasing positive simple functions over $E_{M_{3}, N, a}^{(k)}$ such that $\lim _{i \rightarrow+\infty} \varphi_{i}=l, \varphi_{i}(x)=0$, $\forall x \notin E_{M_{1}, N, a}^{(k)}, \forall i \in \mathbf{N}$, from Beppo Levi theorem we have

$$
\begin{aligned}
\int_{E_{M_{1}, N, a}^{(k)}} l d \lambda_{N, a}^{(k)} & =\lim _{i \rightarrow+\infty} \int_{E_{M_{1}, N, a}^{(k)}} \varphi_{i} d \lambda_{N, a}^{(k)} \\
& =\lim _{i \rightarrow+\infty} \int_{E_{M_{2}, N, b}^{(k)}} \varphi_{i}\left(h_{n}\right)\left|\operatorname{det} h_{n}\right| d \lambda_{N, b}^{(k)} \\
& =\int_{E_{M_{2}, N, b}^{(k)}} l\left(h_{n}\right)\left|\operatorname{det} h_{n}\right| d \lambda_{N, b}^{(k)} \\
& =\lim _{n \rightarrow+\infty} \int_{E_{M_{2}, N, b}^{(k)}} l\left(h_{n}\right)\left|\operatorname{det} h_{n}\right| d \lambda_{N, b}^{(k)} .
\end{aligned}
$$

In particular, the formula (62) is true for any continuous and bounded function $l: E_{M_{3}, N, a}^{(k)} \rightarrow[0,1]$. In this case, let $\left\{f_{n}\right\}_{n \in \mathbf{N}}$ be the sequence of the measurable functions $f_{n}$ : $\left(E_{M_{2}, N, b}^{(k)}, \mathscr{B}\left(E_{M_{2}, N, a}^{(k)}\right)\right) \rightarrow(\mathbf{R}, \mathscr{B})$ given by

$$
f_{n}(x)=l\left(h_{n}(x)\right)\left|\operatorname{det} h_{n}\right|, \quad \forall x \in E_{M_{2}, N, b}^{(k)}, \forall n \in \mathbf{N} .
$$

Since $\operatorname{det} h_{n}=\operatorname{det} A, \forall n \geq m$, we have $\left|f_{n}\right| \leq g$, where $g:\left(E_{M_{2}, N, b}^{(k)}, \mathscr{B}\left(E_{M_{2}, N, a}^{(k)}\right)\right) \rightarrow([0,+\infty), \mathscr{B}([0,+\infty)))$ is the measurable function defined by

$$
g(x)=|\operatorname{det} A|, \quad \forall x \in E_{M_{2}, N, b}^{(k)} .
$$

Moreover

$$
\begin{aligned}
& \int_{E_{M_{2}, N, b}^{(k)}} g d \lambda_{N, b}^{(k)} \\
& =|\operatorname{det} A| \lambda_{N, b}^{(k)}\left(E_{M_{2}, N, b}^{(k)}\right) \\
& =\frac{|\operatorname{det} A|\left(2 M_{2}\right)^{k}}{(2 N)^{k}} \prod_{p>k}\left(\frac{1}{2 N} \operatorname{Leb}\left(\left[-N b_{p}, N b_{p}\right]\right)\right) \\
& =\frac{|\operatorname{det} A| M_{2}^{k}}{N^{k}} \prod_{p>k} b_{p}<+\infty .
\end{aligned}
$$

Moreover, we have $\lim _{n \rightarrow+\infty} h_{n}=A$, and so $\lim _{n \rightarrow+\infty} f_{n}=$ $l(A)|\operatorname{det} A|$; then, from the dominated convergence theorem,

$$
\begin{gathered}
\lim _{n \rightarrow+\infty} \int_{E_{M_{2}, N, b}^{(k)}} l\left(h_{n}\right)\left|\operatorname{det} h_{n}\right| d \lambda_{N, b}^{(k)} \\
=\int_{E_{M_{2}, N, b}^{(k)}} l(A)|\operatorname{det} A| d \lambda_{N, b}^{(k)} .
\end{gathered}
$$

Then, from (62) we have

$$
\int_{E_{M_{1}, N, a}^{(k)}} l d \lambda_{N, a}^{(k)}=\int_{E_{M_{2}, N, b}^{(k)}} l(A)|\operatorname{det} A| d \lambda_{N, b}^{(k)} \text {. }
$$

Let $B=\prod_{p \in I} B_{p} \in \mathscr{B}\left(E_{M_{1}, N, a}^{(k)}\right)$, where $B_{p}=\left(a_{p}, b_{p}\right), \forall p \in$ $I$; moreover, $\forall n \in \mathrm{N}^{*}$, consider the continuous function $l_{n}$ : $E_{M_{3}, N, a}^{(k)} \rightarrow[0,1]$ defined by

$$
l_{n}(x)= \begin{cases}1 & \text { if } x \in \prod_{p \in I}\left(a_{p}+\frac{\delta_{p}}{n}, b_{p}-\frac{\delta_{p}}{n}\right) \\ \frac{\left\|x-x_{2}\right\|}{\left\|x_{1}-x_{2}\right\|} & \text { if } x \in B \backslash \prod_{p \in I}\left(a_{p}+\frac{\delta_{p}}{n}, b_{p}-\frac{\delta_{p}}{n}\right) \\ 0 & \text { if } x \notin B,\end{cases}
$$

where $\delta_{p}=\left(b_{p}-a_{p}\right) / 2, \forall p \in I, x_{1}=r \cap \partial\left(\prod_{p \in I}\left(a_{p}+\right.\right.$ $\left.\left.\left(\delta_{p} / n\right), b_{p}-\left(\delta_{p} / n\right)\right)\right), x_{2}=r \cap \partial B$, where $r$ is the half-line with initial point $\prod_{p \in I}\left(\left(a_{p}+b_{p}\right) / 2\right)$ and containing $x$. Since $\left\{l_{n}\right\}_{n \in \mathbf{N}}$ is an increasing positive sequence such that $\lim _{n \rightarrow+\infty} l_{n}=1_{B}$, from Beppo Levi theorem and (67), we have

$$
\begin{aligned}
\int_{B} d \lambda_{N, a}^{(k)} & =\lim _{n \rightarrow+\infty} \int_{E_{M_{1}, N, a}^{(k)}} l_{n} d \lambda_{N, a}^{(k)} \\
& =\lim _{n \rightarrow+\infty} \int_{E_{M_{2}, N, b}^{(k)}} l_{n}(A)|\operatorname{det} A| d \lambda_{N, b}^{(k)} \\
& =\int_{A^{-1}(B)}|\operatorname{det} A| d \lambda_{N, b}^{(k)} .
\end{aligned}
$$

Moreover, Proposition 28 again implies that the formula (69) is true $\forall B \in \mathscr{B}\left(E_{M_{1}, N, a}^{(k)}\right)$. Consider the measures $\mu$ and $v$ on $\mathscr{B}\left(E_{N, a}^{(k)}\right)$ defined by

$$
\begin{aligned}
& \mu(B)=\int_{B} d \lambda_{N, a}^{(k)}, \\
& v(B)=\int_{A^{-1}(B)}|\operatorname{det} A| d \lambda_{N, b}^{(k)},
\end{aligned}
$$

and set $B_{n}=B \cap E_{n, N, a}^{(k)}, \forall n \in \mathbf{N}^{*}, \forall B \in \mathscr{B}\left(E_{N, a}^{(k)}\right)$. Since $B_{n} \subset B_{n+1}, A^{-1}\left(B_{n}\right) \subset A^{-1}\left(B_{n+1}\right), \bigcup_{n \in \mathbf{N}^{*}} B_{n}=B$, and $\bigcup_{n \in \mathbf{N}^{*}} A^{-1}\left(B_{n}\right)=A^{-1}(B)$, from the continuity property of $\mu$ and $v$ and (69), we have

$$
\begin{aligned}
\int_{B} d \lambda_{N, a}^{(k)} & =\lim _{n \rightarrow+\infty} \int_{B_{n}} d \lambda_{N, a}^{(k)} \\
& =\lim _{n \rightarrow+\infty} \int_{A^{-1}\left(B_{n}\right)}|\operatorname{det} A| d \lambda_{N, b}^{(k)} \\
& =\int_{A^{-1}(B)}|\operatorname{det} A| d \lambda_{N, b}^{(k)} .
\end{aligned}
$$


Then, $\forall D \in \mathscr{B}\left(E_{N, a}^{(k)}\right)$,

$$
\begin{aligned}
\int_{B} 1_{D} d \lambda_{N, a}^{(k)} & =\int_{B \cap D} d \lambda_{N, a}^{(k)}=\int_{A^{-1}(B \cap D)}|\operatorname{det} A| d \lambda_{N, b}^{(k)} \\
& =\int_{A^{-1}(B)} 1_{A^{-1}(D)}|\operatorname{det} A| d \lambda_{N, b}^{(k)} \\
& =\int_{A^{-1}(B)} 1_{D}(A)|\operatorname{det} A| d \lambda_{N, b}^{(k)} .
\end{aligned}
$$

Thus, by proceeding as in the proof of the formula (62), for any measurable function $f:\left(E_{N, a}^{(k)}, \mathscr{B}\left(E_{N, a}^{(k)}\right)\right) \rightarrow$ $([0,+\infty), \mathscr{B}([0,+\infty)))$, we obtain

$$
\int_{B} f d \lambda_{N, a}^{(k)}=\int_{A^{-1}(B)} f(A)|\operatorname{det} A| d \lambda_{N, b}^{(k)} .
$$

Then, if $f:\left(E_{N, a}^{(k)}, \mathscr{B}\left(E_{N, a}^{(k)}\right)\right) \rightarrow(\mathbf{R}, \mathscr{B})$ is a measurable function such that $f^{+}$(or $\left.f^{-}\right)$is $\lambda_{N, a}^{(k)}$-integrable:

$$
\begin{aligned}
\int_{B} f d \lambda_{N, a}^{(k)}= & \int_{B} f^{+} d \lambda_{N, a}^{(k)}-\int_{B} f^{-} d \lambda_{N, a}^{(k)} \\
= & \int_{A^{-1}(B)} f^{+}(A)|\operatorname{det} A| d \lambda_{N, b}^{(k)} \\
& -\int_{A^{-1}(B)} f^{-}(A)|\operatorname{det} A| d \lambda_{N, b}^{(k)} \\
= & \int_{A^{-1}(B)} f(A)|\operatorname{det} A| d \lambda_{N, b}^{(k)} .
\end{aligned}
$$

Finally, suppose that $B \in \mathscr{B}(E)$ and $f:(E, \mathscr{B}(E)) \rightarrow$ $(\mathbf{R}, \mathscr{B})$ is a measurable function such that $f^{+}$(or $f^{-}$) is $\lambda_{N, a}^{(k)}$-integrable; from formula (74), Proposition 25 and definitions of $\lambda_{N, a}^{(k)}$ and $\lambda_{N, b}^{(k)}$ given by (4), we have

$$
\begin{aligned}
\int_{B} f d \lambda_{N, a}^{(k)} & =\int_{B \cap E_{N, a}^{(k)}} f d \lambda_{N, a}^{(k)} \\
& =\int_{A^{-1}\left(B \cap E_{N, a}^{(k)}\right)} f(A)|\operatorname{det} A| d \lambda_{N, b}^{(k)} \\
& =\int_{A^{-1}(B)} f(A)|\operatorname{det} A| d \lambda_{N, b}^{(k)} .
\end{aligned}
$$

\section{Probabilistic Applications}

Definition 30. Let $(\Omega, \mathscr{F}, P)$ be a probability space; a random element $X:(\Omega, \mathscr{F}, P) \rightarrow(E, \mathscr{B}(E))$ is called $\lambda_{N, a}^{(k)}$-continuous if there exists a measurable function $f_{X}:(E, \mathscr{B}(E)) \rightarrow$ $([0,+\infty), \mathscr{B}([0,+\infty)))$ such that, for any $A \in \mathscr{B}(E)$,

$$
P(X \in A)=\int_{A} f_{X} d \lambda_{N, a}^{(k)} .
$$

The function $f_{X}$ is called infinite-dimensional probability density of $X$.
Theorem 31. Let $A=\left(a_{i j}\right)_{i, j \in I}: E \rightarrow E$ be a biunique and linear $(m, \sigma)$-standard function, let $a=\left(a_{n}: n \in I\right) \in\left(\mathbf{R}^{+}\right)^{I}$ be such that $\prod_{n \in I} a_{n} \in \mathbf{R}^{+}$, and let $b \in\left(\mathbf{R}^{+}\right)^{I}$ be the sequence defined by Proposition 25. Then, for any $k \in \mathbf{N}, k \geq m$, for any $N \in \mathbf{R}^{+}$, and for any $\lambda_{N, b}^{(k)}$-continuous random element $X:(\Omega, \mathscr{F}, P) \rightarrow(E, \mathscr{B}(E))$, the random element $T=A \circ X:$ $(\Omega, \mathscr{F}, P) \rightarrow(E, \mathscr{B}(E))$ is $\lambda_{N, a}^{(k)}$-continuous and one has

$$
f_{T}(t)=f_{X}\left(A^{-1}(t)\right)\left|\operatorname{det} A^{-1}\right|, \quad \forall t \in E .
$$

Proof. $\forall B \in \mathscr{B}(E)$, we have

$$
\begin{aligned}
& P(T \in B) \\
& =E\left[1_{B}(T)\right]=E\left[1_{B}(A(X))\right] \\
& =\int_{E} 1_{B}(A(x)) f_{X}(x) d \lambda_{N, b}^{(k)}(x) \\
& =\int_{A^{-1}(B)} f_{X}\left(A^{-1}(A(x))\right)\left|\operatorname{det} A^{-1}\right||\operatorname{det} A| d \lambda_{N, b}^{(k)}(x) \\
& =\int_{B} f_{X}\left(A^{-1}(t)\right)\left|\operatorname{det} A^{-1}\right| d \lambda_{N, a}^{(k)}(t) \text { (from Theorem 29). }
\end{aligned}
$$

\section{Problems for Further Study}

A natural extension of this paper is the generalization of Theorem 29 by considering the measurable and $C^{1}$-invertible functions $A: E \rightarrow E$. As in the finite case, we can define the infinite-dimensional Jacobian matrix of these functions and the determinant of this Jacobian, if it is a $(m, \sigma)$-standard matrix.

Moreover, from Definition 30 and Theorem 31, in the probabilistic context it is possible to introduce many random elements that generalize the well-known continuous random vectors in $\mathbf{R}^{m}$ (e.g., the Gaussian random elements in $E$ defined by the $(m, \sigma)$-standard matrices) and to develop a theory and some applications in the statistical inference.

In particular, as we point out in the introduction, we can generalize the paper [4] by considering the recursion $\left\{X_{n}\right\}_{n \in \mathbf{N}}$ on $\prod_{i \in \mathbf{N}^{*}}[0, p)$ defined by

$$
X_{n+1}=A X_{n}+B_{n} \quad(\bmod p),
$$

where $X_{0}=x_{0} \in E, A$ is a $(m, \sigma)$-standard matrix, $p \in \mathbf{R}^{+}$, and $\left\{B_{n}\right\}_{n \in \mathbf{N}}$ is a sequence of independent and identically distributed random elements on $E$. Our target is to prove that, with some assumptions on the law of $B_{n}$, the sequence $\left\{X_{n}\right\}_{n \in \mathbf{N}}$ converges with geometric rate to a random element with law $\left.\bigotimes_{i \in \mathbf{N}^{*}}(1 / p) \operatorname{Leb}\right|_{\mathscr{B}([0, p))}$. Moreover, we wish to quantify the rate of convergence in terms of $A, p, m$ and the law of $B_{n}$ and to prove that if $A$ has an eigenvalue that is a root of 1 , then $O\left(p^{2}\right)$ steps are necessary to achieve randomness. We hope to develop these ideas in a further paper. 


\section{Conflict of Interests}

The author declares that there is no conflict of interests regarding the publication of this paper.

\section{Acknowledgment}

The author is grateful to Professor Aljosa Volcic for his fruitful suggestions in writing this paper.

\section{References}

[1] R. Léandre, "Lebesgue measure in infinite dimension as an infinite-dimensional distribution," Journal of Mathematical Sciences, vol. 159, pp. 833-836, 2009.

[2] N. Tsilevich, A. Vershik, and M. Yor, "An infinite-dimensional analogue of the Lebesgue measure and distinguished properties of the gamma process," Journal of Functional Analysis, vol. 185, no. 1, pp. 274-296, 2001.

[3] R. Baker, "“Lebesgue measure" on $\mathbf{R}^{\infty}$," Proceedings of the American Mathematical Society, vol. 113, no. 4, pp. 1023-1029, 1991.

[4] C. Asci, "Convergence in total variation of an affine random recursion in $[0, p)^{k}$ to a uniform random vector," Markov Processes and Related Fields, vol. 19, no. 1, pp. 125-140, 2013.

[5] L. Accardi, O. G. Smolyanov, and M. O. Smolyanova, "Change of variable formulas for in.nite-dimensional distributions," Mathematical Notes, vol. 60, pp. 212-215, 1996.

[6] M. M. Rao, Measure Theory and Integration, Pure and Applied Mathematics (New York), John Wiley \& Sons, New York, NY, USA, 1987.

[7] S. Lang, Linear Algebra, Undergraduate Texts in Mathematics, Springer, New York, NY, USA, 3rd edition, 1987.

[8] J. Weidmann, Linear Operators in Hilbert Spaces, vol. 68 of Graduate Texts in Mathematics, Springer, New York, NY, USA, 1980, Translated from the German by Joseph Szücs.

[9] P. Billingsley, Probability and Measure, Wiley Series in Probability and Mathematical Statistics, John Wiley \& Sons, New York, NY, USA, 3rd edition, 1995.

[10] S. Lang, Analysis II, Addison-Wesley, Reading, Mass, USA, 1969. 


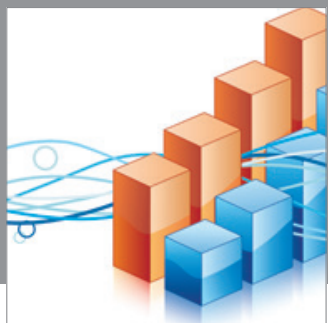

Advances in

Operations Research

mansans

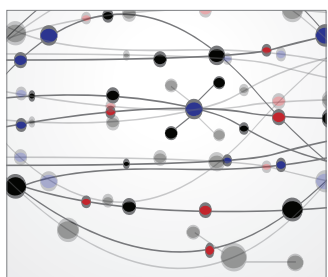

The Scientific World Journal
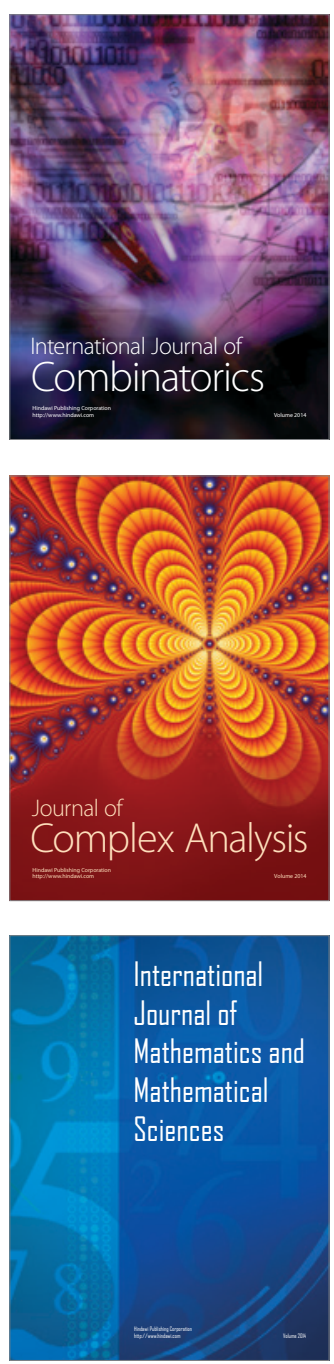
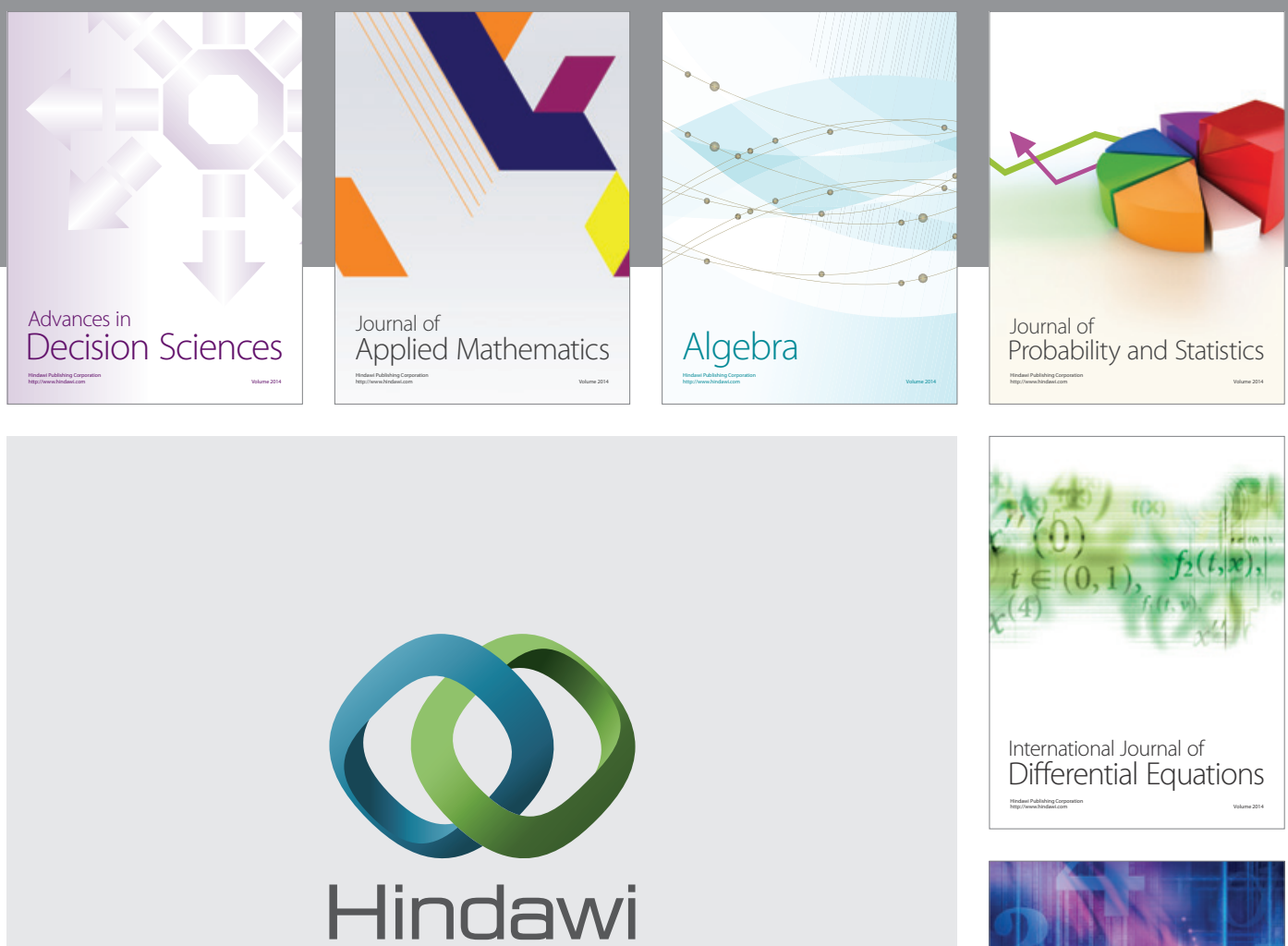

Submit your manuscripts at http://www.hindawi.com
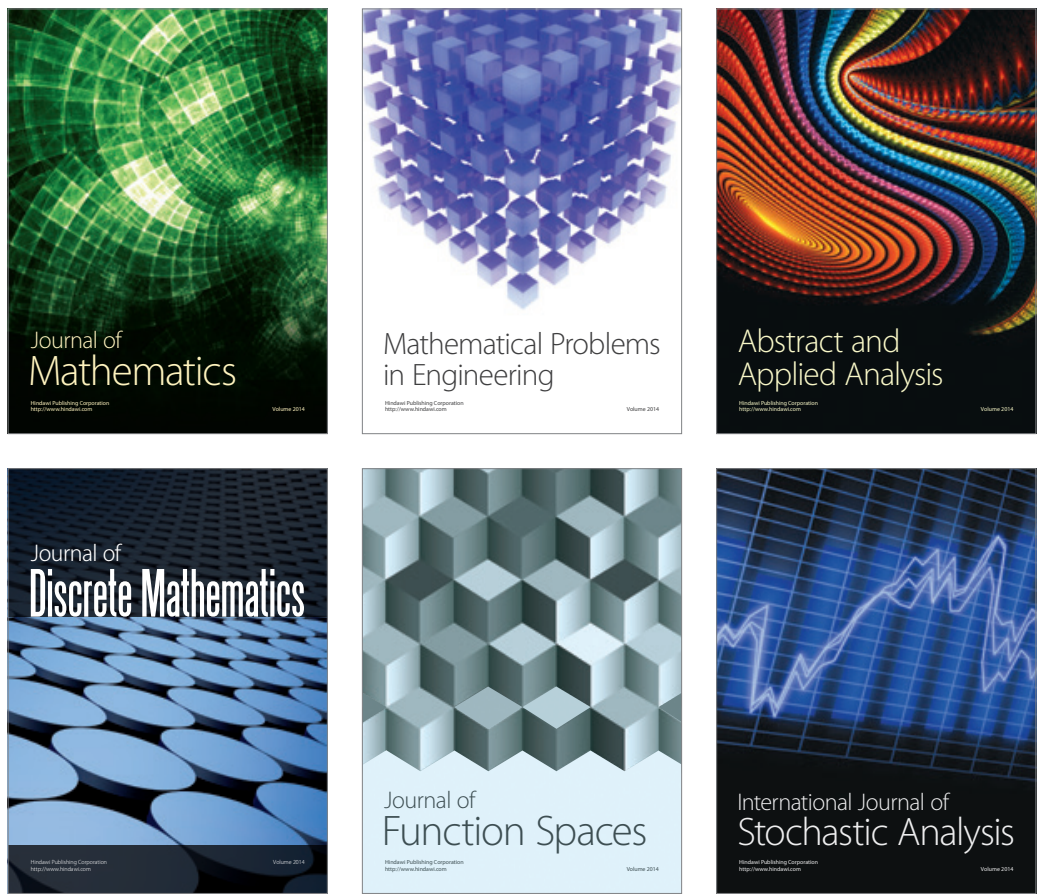

Journal of

Function Spaces

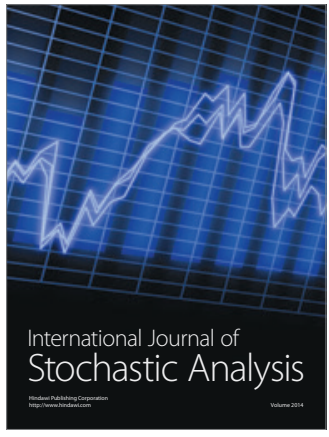

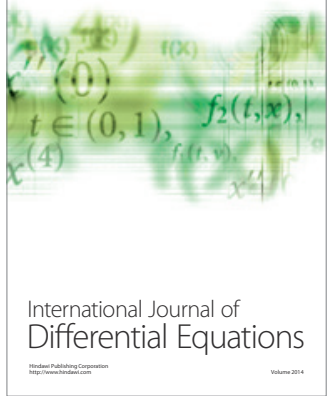
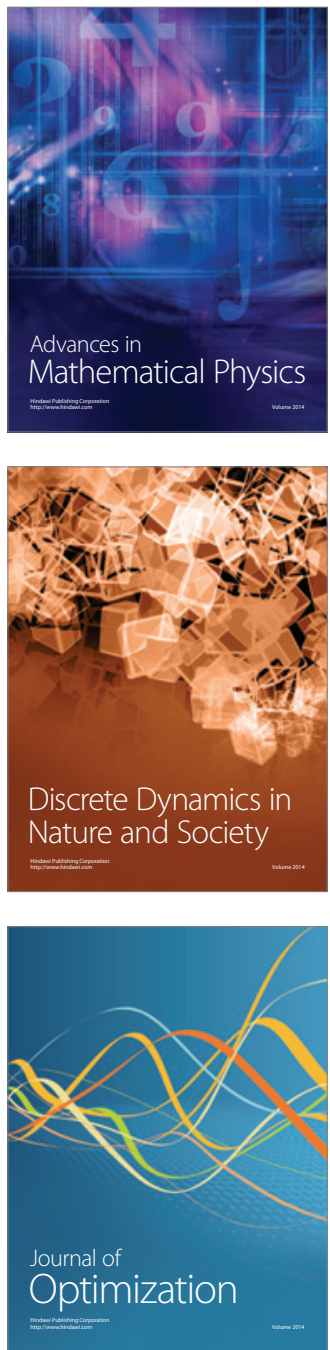